\title{
Intelligent Alarm System of Remote Monitoring Based on Image Processing
}

\author{
Xiaoyu Zhang \\ Zhengzhou Institute of Aeronautic Industry Management, Zhengzhou 450015, China \\ xiaoyu123@qq.com
}

Key Words: remote monitoring of intelligent alarm, monitoring of image processing, Skynet project.

\begin{abstract}
This paper makes discussion on reasonable application of modern image processing technology to improve remote monitoring system of intelligent alarm in the intelligent alarm remote monitoring system, it maximally realizes organic combination of civil air defense, physical protection, technical protection of alarm supervision and management, it can enhance supervision and management efficiency of alarm as well as meanings of technical level and main problems of application.
\end{abstract}

\section{State and development trend of image processing monitoring}

Video monitoring system is one kind of comprehensive system with stronger prevention capacity. Video monitoring is applied in many fields by perceptual intuition, convenience, and rich information content. In the end of 1990s, the appearance and development of multi-media technology, video compression coding technology and network communication technology, as well as quick emergence of image processing monitoring system, it is mainly video monitoring system by using image and video equipment as core as well as video monitoring system by using embedded video Webs server as core. The foreign countries have accumulated plenty of experience in aspects such as road, public security, public community, large-scale buildings of sea, air and port etc; they are gradually applying new development in computer technology, electronic information technology into intelligent monitoring. Because of historical reason, application of security and monitoring technology of China started late. In the middle of the last 1990s, China had stated application of intelligent monitoring by large-scale. In the intelligent monitoring with larger population and quick economic development in south of China, due to complication in social security, projects such as street with electronic monitoring, monitoring community and video monitoring in large-scale public areas are appearing one after another, they are mainly used in intelligent traffic management system and large-scale public facility. It has gradually formed relatively complete intelligent monitoring system of management system by using video monitoring as main body and by integrating every aspect of intelligence.

In recent years, with great promotion in safety engineering, there are many intelligent monitoring in China form industrial fields cross public security, traffic, culture etc. Monitoring net of image processing technology, in the intelligent security prevention and social management practice, it is displaying more and more important effect. It can be predicted that with the gradual mature in video monitoring system, intelligent monitoring system is developing towards intelligence and networking.

\section{Introduction to remote monitoring system of intelligent alarm in China}

Remote monitoring system of intelligent alarm makes networking on independent fair disaster automatic alarm system in balancing by modern communication network, informatization application system makes real-time monitoring on fair alarm condition, centralized management on alarm facility by comprehensively applying geological information system, image processing and monitoring etc in the monitoring center. There are many developed areas in abroad have established intelligent remote monitoring on fair automatic alarm net. It makes networking on control center of fair automatic 
alarm system in important protection units(such as hospital, kindergarten, nursing hone, gas station, dense-population area) and alarm remote monitoring center, intelligent monitoring center will be responsible for network management and warning disposal, it will contact warning unit personnel and warning command center.

At present, most automatic alarm systems applied in China generally adopts site alarm, regional automatic alarm system with concentrated and scattered control ways, centralized automatic alarm and automatic alarm of control center. The composition part of intelligent alarm remote monitoring system mainly includes intelligent remote monitoring center, networking user and user transmission device, alarm acceptance system, information inquiry system and user service and management system etc. The third party will make medium operation unit for intelligent emote monitoring center, it will allocate professional technicians to make duty of 24 hours, it will make timely management on alarm acceptance system. User transmission device is installed in the important fair prevention units (networking user) connected with alarm server in units to receive alarm and fault information. The alarm department can check all the alarm and fault information conditions of all the units in the whole networking jurisdictions areas by information search system, networking users can check work state of alarm server, alarm and fault conditions of alarm facility in building of unit by user service and management system. This system is mainly stressing remote monitoring and alarm disposal of automatic alarm system equipment. It has not completely displayed effect of image processing; there is still limitation in application.

\section{Application of monitoring in image processing}

Overall structure of system. Wireless image processing and transmission system are realized on the basis of $3 \mathrm{G}$ wireless communication application, we can convert and compress the simulation signal collected by image processing by $3 \mathrm{G}$ network into digital signal, and then send to server to make parallel indication. Meanwhile, the server can send out audio frequency, image processing as well as control signal on camera and focus to make command and operate camera according to requirement. All of this needs to be realized in $3 G$ network.

The system includes front end of image processing and collection, receive and indication end of network transmission and image processing. The computer of front terminal mainly includes computer, microphone, and sound box. Computer is mainly used control and collection equipment to receive and collect information, and then sends to receive terminal of image processing after coding. Meanwhile it will receive and broadcast the returned information. The receive terminal mainly responsible for receiving and indicating the information send by the front terminal, collect video and audio frequency to send to front terminal. From the procedure, the function of collection terminal and receive terminal are equal, but convenient for distinction, we regard it as front terminal and receive terminal.

The system frame is indicated by Fig. 1, we can see that system collection front terminal and receive terminal of plenty of image processing are connected by Internet, but not each receive terminal will receive image processing collected from front terminal of image processing, some receive terminal only receive one or several image processing collected by front terminal, in order to solve this problem, we establish one central data base, of which it stores corresponding relations of every user, on user entry., it will verify login information with central data base, the service procedure of central data base will send user information and state to users, and then it will be indicated in the user list table that can carry out image processing and communication, then user can choose one or several users to make image processing and communication.

Except for the above parts used in audio frequency transmission and indication of image processing and indication, system also includes some self-control mechanism, it is used in required information on operation of synchronization system. Of which, it includes data base search, updating and information communication mechanism. The data base adopts independent central data base, 

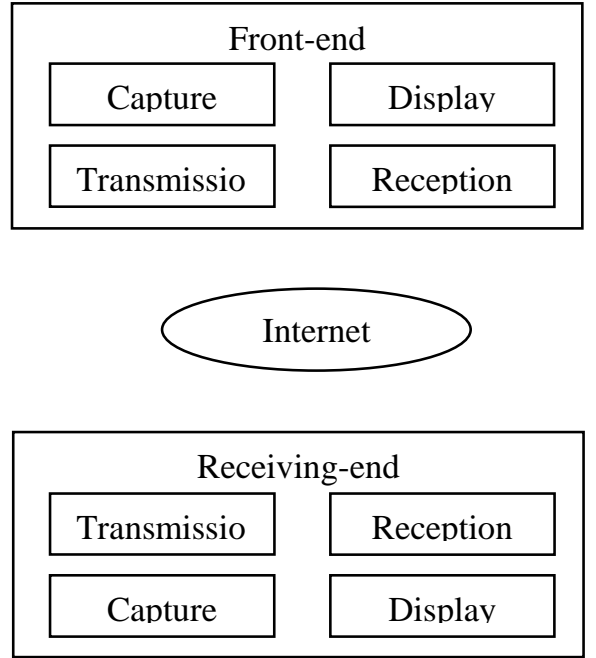

Fig. 1 System frame diagram

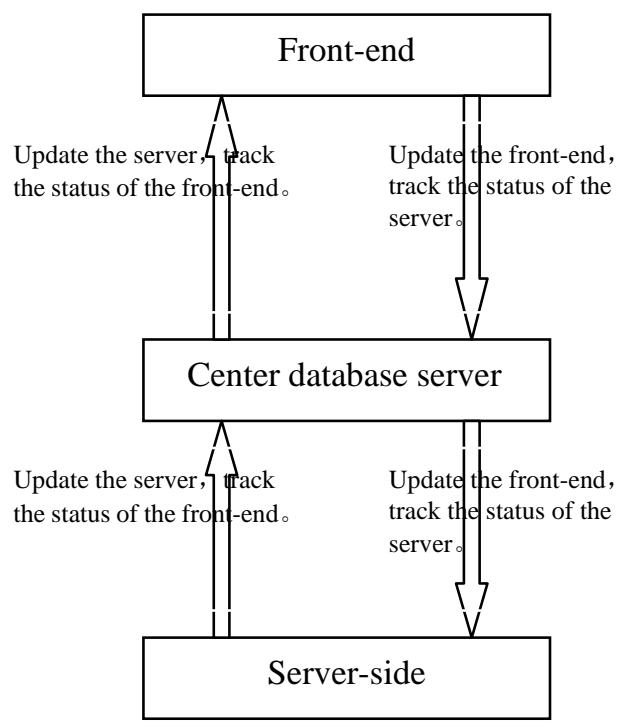

Fig. 2 Information communication frame

There is one server responsible for search and updating, the procedure of front terminal and service terminal will communicate with central server, search information or send out command of operation data by information communication mechanism. Its structure diagram is indicated by Fig. 2 .

Technology chosen by system. Before introduction to sub-module system, firstly we need to understand the circulation procedure of image processing frequency then we can master effect and design aim of each function module. Here we only simply introduce the image processing and circulation procedure of one-way for convenient explanation. It is indicated by Fig. 3, firstly camera will collect image processing, the collected image processing stats image processing coding after captured by computer, aim of image processing coding is to well make network transmission, the image processing data undergone coding is sent to $3 \mathrm{G}$ base station by $3 \mathrm{G}$ network card and connected to Internet by $3 \mathrm{G}$ base station link, then made transmission in Internet. The image processing data in Internet is captured by targeted computer; computer will start coding and indication after capturing image processing.

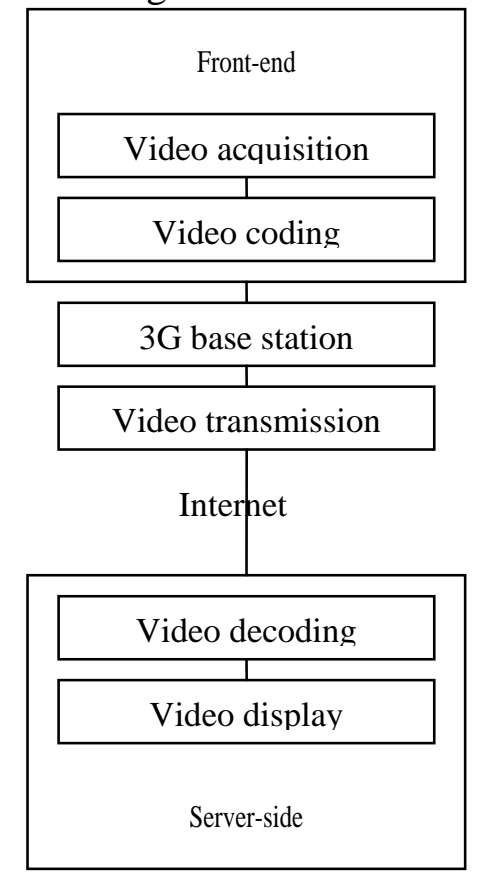

Fig. 3 Circulation process on frequency data of image processing
RTP/RPCP （application layer protocol）

TCP/UDP （transport layer protocol）

IP (network layer protocol)

MAC (physical layer protocol)

Fig. 4 Typical internet protocol model 
After learning circulation process of image processing, we can start understanding technical route of every module.

Application of real-time multi-media needs timely information transmission; it allows some loss data to realize this target. For example, data loss in frequency application, it may cause damage in some frequency, while the second frequency data can make proper error concealment algorithm according to the loss data of the first data packet. The transmission of TCP standardizes application of RTP, but it is different from RTP application, because TCP advocates the reliability of exceeding timeliness. On the contrary, most RTP realization are established on UDP. There are also some other special designs used in other transfer protocol SCTP and DCCP of multi-media dialogue. However, until 2010, they had not got universal application.

RTCP is the service control protocol of RTP. RTCP provides statistics data and control information of RTP. It is used for controlling delivery and packing of multi-media data in RTP, but it dose not transmit any media stream. RTCP information is sent to the next higher odd port in the even UDP port of sending RTP. The basic function of RTCP is to provide media distribution of service quality to send statistics information stream to participators of multi-media dialogue. The position of RTP protocol and RTCP protocol in Internet protocol suite is indicated by Fig. 4.

Such as indicated by diagram 3-4, RTP and RTCP protocols belong to protocol of application level, it is specially used for transmission of stream media. This system adopts RTP and RTCP as transmission protocol as image processing.

Design on internal communication mechanism of system. This system adopts unique information receive and send, event trigger mechanism to enhance real-time and degree of automation of system. It defines one frame for information and event according to the required information and possible conditions in the transmission process; it can freely add information and event frame.

This system adopts solutions of low cost, its cost is 1.5 times of communication equipment cost of image processing at present, it can provide mobile image processing and communication service of the same quality even higher quality. Firstly, on choice of internet, we can adopt high-speed 3G network but not satellite network of high cost, which reduces much more system cost, on the choice of image processing equipment, we choose equipment that can be bought in market and then continue to make development on its base, but not specially develop one set of image processing equipment, which greatly reduces our cost.

\section{Acknowledgements}

I would like to express my gratitude to all those who have helped me during the writing of this thesis. I gratefully acknowledge the help of my supervisor Professor Liu. I do appreciate his patience, encouragement and professional instructions during my thesis writing. Also, I world like to thank $\mathrm{Mr}$ Yang, who kindly gave me a hand when I was making the questionnaire. Last my gratitude extends to my family who have assisting, supporting and caring for me all of my life.

\section{References}

[1] W.P. Liu, X.C. Yang, J.Z. Wang and D. Meng: The remote video monitoring alam system based on GPRS, Wireless Communication Technology, (2008)No.2. (In Chinese)

[2] Pan Gou: Design and implementation of remove web video surveillance intelligent alarm system, (MS., Southwest jiaotong university, China 2009), p.1-3. (In Chinese)

[3] S.C. Yu: The research of remote unattended image recording alarm system based on the $3 G$ network(MS., Changchun University of Science and Technology, China 2012), p. 33-36 (In Chinese)

[4] W.T. Shao: Development of intelligent of embedded video surveillance alarm system based on B/S model(MS., Northeastern University, China 2010), p. 64-67.(In Chinese) 